\title{
Archaïsmes religieux dans les tragédies de Sénèque
}

Marie Delcourt

\section{Citer ce document / Cite this document :}

Delcourt Marie. Archaïsmes religieux dans les tragédies de Sénèque. In: Revue belge de philologie et d'histoire, tome 42, fasc.

1, 1964. Antiquité — Oudheid. pp. 74-90;

doi : 10.3406/rbph.1964.2505

http://www.persee.fr/doc/rbph_0035-0818_1964_num_42_1_2505

Document généré le 28/06/2017 


\section{ARCHAISMES RELIGIEUX DANS LES TRAGEDIES DE SENEQUE}

Quelques poètes tardifs ont un sens mythologique si exact que lorsqu'ils développent une légende c'est, si l'on peut ainsi s'exprimer, dans la bonne direction. Les détails qu'ils empruntent, soit à leur imagination, soit à des traditions divergentes, se trouvent préciser heureusement la signification primitive de l'histoire, signification qu'eux-mêmes ne se sont pas souciés d'approfondir (ainsi que nous le faisons à grand renfort de rapprochements ingénieux), mais sur laquelle une sorte d'instinct semble les avoir éclairés. Les historiens des religions ont souvent l'occasion d'admirer une telle intuition chez Ovide, chez Nonnos. Ils s'étonnent davantage lorsqu'ils la découvrent à l'œuvre chez Sénèque.

Un poète constamment tendu et emphatique, dont l'outrance et l'hyperbole sont les moyens favoris, comment s'attendre qu'il revienne au sens premier d'une légende par delà des interprétations plus rationalisées? C'est cependant ce que Charles Fontinoy a fort bien démontıé à propos de l'égorgement de Polyxène sur la tombe d'Achille dans les Troyennes $\left(^{(}\right)$. Il y décèle le sacrifice, attesté dans le folklore slave, où il fut certainement réel avant de devenir symbolique, d'une jeune fille au moment des funérailles d'un jeune célibataire. Ce n'est pas exactement l'immolation de la suttee : celle-ci suit le mari dont elle est la propriété, tandis que l'innuptus reçoit la vie de la jeune fille comme une compensation à tout ce dont le frustre un décès prématuré. La victime est mariée au mort.

Cela n'apparaît guère dans Hécube. La reine désigne bien sa fille

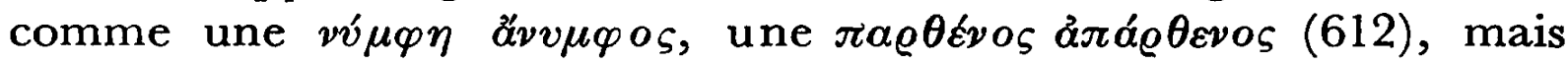
$M$. Fontinoy juge avec raison qu'il ne faut pas trop presser le sens

(1) Le sacrifice nuptial de Polyxène dans Antiquité classi que, t. XIX (1950), p. 383 sqq. 
de cet oxymoron. J'attacherais volontiers plus d'importance au refus d'Ulysse, qui, lorsque Hécube propose de se substituer à sa fille, lui répond que ce n'est pas elle, « la vieille», qu'exige l'ombre d'Achille, mais bien Polyxène (389), ce qui indique que le mort a choisi son geras. Considérons aussi le geste de Polyxène qui, devant Néoptolème qui va la frapper, "se déchire la robe de l'épaule au nombril» (559), suggérant une mariée qui se dévoile, alors que le couteau doit lui trancher la gorge : pur symbole livré tel quel à l'auditeur qui n'a pas le temps d'en dégager explicitement la signification. Euripide n'a pas aidé Sénèque à redécouvrir la valeur archaïque du thème.

Si Sénèque a connu les versions tardives qui parlent de relations amoureuses entre Achille vivant et la princesse troyenne, il a eu le bon goût de n'en rien laisser affleurer dans sa tragédie. Le fantôme d'Achille exige simplement :

Desponsa nostris cineribus Polyxena

Pyrrhi manu mactetur (195-6),

sur quoi Agamemnon proteste :

Facinus atrox caedis ut thalamos vocent non patiar (289-90),

mais Calchas l'oblige d'accorder le praemium exigé par le héros :

Pyrrhus parenti conjugem tradat suo (364).

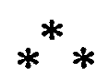

Le même Calchas annonce aussitôt qu'un autre sang est exigé : du haut d'une tour, la seule qui soit encore debout au milieu des ruines de Troie, le fils d'Hector doit être précipité.

Euripide a fait du meurtre d'Astyanax l'épisode le plus pathétique des Troyennes. Comme dans l'Iliou Persis, c'est Ulysse qui décide la mise à mort. La Petite Iliade jette tout l'odieux sur Neoptolème qui saisit l'enfant par un pied, l'arrache du sein de sa nourrice et le lance du haut de la tour. Les variantes portent sur les détails de l'exécution. Aucun poète ne semble avoir donné une justification différente de celle qu'allègue Ulysse dans les Troyennes grecques : «Il ne faut pas que vive le fils d'un tel héros" (723). "Ici gît un enfant que les 
Grecs ont tué parce qu'ils le craignaient. Honte pour la Hellade qu'une telle inscıiption !» dira Hécube (1190-1).

Cette prudence à long terme dut paraître une raison insuffisante. On la renforça d'un nouvel argument: les destins auraient prédit que l'enfant, s'il vivait, vengerait sa patrie et ses parents (Servius, Enéide, II, 457). Mais cela même ne dépasse pas la raison d'État.

En réalité, l'immolation d'Astyanax recouvre le sacrifice rituel d'un enfant à la dédicace d'une nouvelle cité. Les contes et légendes de tous les pays racontent des Bauopfer de ce genre, dont la réalité est attestée par des squelettes de jeunes filles, d'enfants, d'animaux, dans des fondations de monuments, des ponts par exemple. C'étaient probablement, à l'époque historique, de pauvres êtres sans défense.

Le sacrifice du fils premier-né avait à l'origine une valeur toute differente, qui apparaît nettement dans les chapitres de l'Exode où il est codifié $\left({ }^{1}\right)$. Les Israélites devaient à Jéhovah tout ce qui, pour naître, avait dû «ouvrir la matrice». Seuls les petits de l'homme et de l'âne étaient épargnés, à condition qu'un agneau fût immolé pour leur rachat. L'Eternel avait dit: «Consacre-moi tout premierné ; il m'appartient» (XIII, 1) ; «l'on ne se présentera point les mains vides devant ma face» (XXXIV, 19). L'immolation atteste le droit du Seigneur sur toute créature ; qu'il laisse vivre la géniture pourvu que les prémices lui en aient été offertes.

On sentit bientôt le besoin d'une justification supplémentaire. Le prophète Michée se demande (VI, 7) :

«Donnerai-je pour mes transgressions mon fils premier-né?

pour le péché de mon âme le fruit de mes entrailles»?

Surtout, on surchargea l'offrande du premier-né des valeurs du Bauopfer. "Le roi de Moab, voyant qu'il avait le dessous dans la mêlée, prit son fils premier-né qui devait régner à sa place et l'offrit en holocauste sur la muraille ». Une grande indignation, ajoute le narıateur, s'empara d'Israël (II Rois, III, 27). Le peuple élu, en effet, ne reconnaissait plus là le sentiment qui avait dicté les prescriptions de l'Exode. Eusèbe rapporte cependant un passage de Philon de Byblos signalant comme une ancienne coutume attestée chez les

(1) Cf. J. G. Frazer, The dying god, chap. VI : Sacrifice of the king's son. 
Juifs : « Dans un grand danger, le maître d'un pays donne à la mort son fils bien-aimé, comme une rançon offerte aux puissances hostiles; le sacrifice de ces enfants était entouré de rites religieux. Ainsi Cronos, que les Phéniciens nomment El, étant roi du pays et ayant un fils unique nommé Jéoud, l'habilla des vêtements royaux et le sacrifia sur un autel pendant une guerre où le pays était en grand danger» (1). Les Hébreux cependant n'ont accepté le sacrifice du prince royal que sous des formes atténuées. Jéhovah épargne Isaac après avoir demandé sa vie afin d'éprouver Abraham (Genèse, XXII) ; Jephté promet, s'il est vainqueur des Ammonites, d'offrir en holocauste la première personne qui viendra à sa rencontre et ce sera sa fille, son unique enfant (Juges, XI, 30). Saül après la bataille de Mikmash condamne Jonathas pour avoir violé une interdiction alimentaire; le peuple toutefois empêche le père de mettre à mort le jeune héros (I Samuel, XIV, 34 sqq.). On devine sous ce récit une immolation analogue à celle du prince de Moab, faite ou promise à Jéhovah comme rançon de la victoire. Le mobile et le dénouement ont été ensemble modifiés.

Des altérations analogues apparaissent dans les versions classiques du sacr ifice du prince. Dans l'histoire des Athamantides (Hérod. VII, 197), il est présenté comme un châtiment. Le plus souvent il sert à acheter une victoire, et un oracle lui sert d'excuse. C'est ainsi qu'Érechthée et Léos immolent leurs filles pour le salut d'Athènes, qu'Agamemnon égorge la sienne afin que la flotte parvienne en Troade, du moins dans la tradition suivie par Eschyle et Racine; car ni les cycliques, ni Sophocle ni Euripide n'ont plus osé prêter à Artémis une exigence gratuite: ils ont voulu qu'Agamemnon fût contraint par un vœu imprudent ou par une faute commise envers la déesse. Des historiens candides se sont demandé si le sang des filles vierges avait en soi une efficacité religieuse. Étrange erreur : la devotio exemplaire, celle de Codrus (Paus. VII, 25, 2), celle de Mettius Curtius (Tite-Live, VII, 6), est celle d'un homme fait : le roi lui-même ou ce qui lui ressemble le plus, un prince revêtu des

(1) Eusèbe, Prép. évang., I, 10, p. 40c. Philon dans son livre sur les Juifs expose une théologie evhémérisée où Cronos-El divinisé après sa mort et devenant l'étoile Saturne sacrifie le fils qu'il a eu d'une nymphe. Renseignement répété IV, 16, p. 156 d. 
vêtements royaux, ainsi que le précise Philon. Mais les dieux, quels qu'ils soient, n'exigent jamais des hommes que ce que ceux-ci sont prêts à accorder : leurs filles plutôt que leurs garçons. Idoménée ne sacrifie son fils que parce qu'il a fait, à l aveugle, le même vœu imprudent que Jephté; encore en est-il puni $\left(^{(1)}\right.$. Créon refuse de donner son fils Ménécée que l'oracle exige pour le salut de Thèbes (Eur. Phén., 961), tandis qu'Agamemnon égorge Iphigénie sans que Mycènes soit en péril.

Alors qu'une tradition qui semble avoir été unanime présentait le meurtre d'Astyanax comme un acte purement politique, Sénèque lui a restitué une valeur religieuse. Non, à vrai dire, celle que les parallèles grecs et orientaux nous permettent de deviner : cette signification primitive, il ne pouvait la retrouver, comme il l'a fait pour l'immolation de Polyxène. Une tradition séculaire avait déplacé la mort de l'enfant : au lieu qu'elle eût figuré, soit à la construction de la ville, soit au moment où le salut de Troie était encore concevable, Sénèque l'a trouvée associée à la ruine finale, quand aucune rançon, fût-ce le cadavre du dernier Priamide, ne pouvait plus sauver le royaume condamné. Il lui a du moins gardé son caractère essentiel : monnaie d'échange pour une faveur divine :

Quem fata quaerunt, turre de summa cadat

Priami nepos Hectoreus et letum oppetat.

Tum mille velis impleat classis freta.

Ainsi ordonne Calchas (368-370), donnant au sacrifice d'Astyanax le même sens qu'Euripide accorde, faute d'en sentir encore l'archaïque caractère nuptial, à celui de Polyxène. L'Achille grec, irrité qu'on lui cût refusé son geras, se comportait comme Artémis à Aulis et exigeait du sang humain. Au lieu d'un seul obstacle au

(1) Cf. J. G. Frazer, Apollodorus Library, appendix XII, the vow of Idomeneus. Le second mythographe du Vatican rend l'histoire plus admissible en disant que le roi n'a sacrifié, comme Jephté, qu'une fille.-R. Crahay me signale dans les Argonautes duPacifique occidental, de Bronislaw Maxinovski, l'épisode de la pirogue immobilisée par la pieuvre jusqu'au moment où l'équipage se décide à sacrifier l'un des petits garçons qui l'accompagne. "Un indigène à qui je demandais pourquoi on ne sacrifiait pas un adulte me répondit : Un adulte ne voudrait pas; un enfant n'a pas a dire son avis : on le prend de force» (p. 296). Aucun acte religieux n'est totalement exempt de composantes de cet ordre. Il est bon de s'en souvenir. 
départ de la flotte Sénèque en donne deux : la colère d'Achille et l'exigence d'une puissance désignée simplement comme fata ou sors.

Ulysse vient annoncer à Andromaque la décision des chefs. Son discours joue curieusement sur deux tons différents. Durae minister sortis, il déclare que les destins exigent ce prix pour permettre aux Grecs de rentrer chez eux (524-528). Puis, de l'argument religieux il passe à la raison d'État : ses amis n'entendent pas laisser derrière eux un futur Hector (550). Cet argument ne sera plus évoqué dans le reste de la pièce et Sénèque aurait pu le laisser de côté. Mais comment le supprimer quand celui qui parle est le politique par excellence, cet Ulysse qui, chez Euripide, est l'instigateur de la condamnation - chez Sénèque tout vient de Calchas - et dont Talthybios se borne à répéter les ordres? Le poète a été entraîné par la psychologie de son personnage. Le discours d'Ulysse, commencé sur une note religieuse, est ensuite purement laïc. Lorsqu'Andromaque lui raconte que l'enfant est mort, il feint de la croire et s'écrie :

\section{Stirpe sublata Hectoris \\ Solidam pacem laetus ad Danaos feram (605-6).}

Et Andromaque, faisant sortir l'enfant du tombeau, jette avec mépris à la tête du Grec, comme Hécube dans la pièce d'Euripide,

$$
\begin{aligned}
& \text { Hic, hic est terror, Ulixe, } \\
& \text { Mille carinis (706-7). }
\end{aligned}
$$

Mais à partir de ce moment, tout redevient purement religieux. Ulysse fait monter la petite victime au sommet du seul créneau qui reste de l'enceinte,

Nota quondam turris et muri decus (1075),

cette tour unique dont le dernier Priamide est comme le symbole. Ulysse joue le rôle du prêtre, prononce les paroles rituelles et les prières :

\section{Verba fatidici et preces}

Concipit Ulixes vatis et saevos ciet Ad sacra superos (1100-2).

A ce moment Astyanax s'élance dans le vide par le mouvement même qui est celui de la devotio, , semblable à Ménécée qui s'égorge au faite des remparts ( $\dot{\xi} \xi \xi \xi \alpha \hat{\varepsilon} \lambda \xi \varepsilon \omega \nu$ äx@mv, Phéniciennes, 1009), d'où 
son sang coule dans l'antre du dragon. Astyanax ne peut plus rien sauver et sa devotio est sans objet. Du moins Sénèque en a-t-il retrouvé la couleur religieuse primitive.

Il est le seul auteur qui montre Astyanax se sacrifiant volontairement. Pour cela, il a dû faire un garçonnet du $\beta \varrho \varepsilon ́ p o \varsigma$ de Leschès que Pyrrhos arrache du sein de sa nourrice, le même qui, chez Euripide, reçoit comme lit funèbre le bouclier de son père. Les peintres ont volontiers $r$ eprésenté un Astyanax adolescent : il est tel sur le vase où Brygos a groupé les épisodes caractéristiques de la prise d'Ilion. Ailleurs, Pyrrhos tient le bébé et le jette, non point du haut d'une tour, mais sur l'autel où Priam est en suppliant $\left(^{1}\right)$. Il ne semble pas que cela vienne d'une source littéraire. Les peintres ont simplement voulu montrer tout d'un coup Pyrrhos meurtrier de Priam (d'après Arctinos) et d'Astyanax (d'après Leschès).

Mais comment ne pas remarquer que Priam est constamment évoqué à la fin des Troyennes latines?

Le messager rappelle que la seule tour subsistante est celle où Priam se tenait pour suivre la bataille (1068), qu'il y emmenait son petit-fils pour le faire assister aux exploits de son père. L'enfant est désigné (1090) comme petit-fils de Priam, non comme fils d'Hector. Enfin il se jette in media Priami regna (1113), formule qui aurait tout son sens si sa devotio devait assurer la solidité des domaines de Priam - et tel en effet devait être le sens premier du xite - mais dont on voit mal la valeur dans l'épisode tel qu'il nous est présenté. Nous avons là un curieux retournement chronologique. La tour épargnée, dirait-on, doit sa permanence, non pas à un sacrifice ancien, comme il serait normal, mais au sacrifice auquel elle était destinée lorsque tout serait perdu pour Troie : elle ne survivrait à toutes les autres que pour voir tomber le dernier prince troyen au milieu de ce qui avait été le regnum Priami. La devotio de l'enfant prend toute sa grandeur du fait d'êtı e sans espoir.

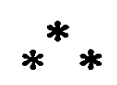

Andromaque tente de sauver son fils en le cachant dans le mausolée d'Hector et en le déclarant mort. Ulysse feint de la croire.

(1) Carl Ronert, Bild und Lied, pp. 29 et 229. 
Mais dit-il, les remparts exigent un lustrale sacrum (634) et, à défaut de l'enfant, les bateaux ne pourront partir que si les cendres d'Hector sont jetées dans la mer et si le tombeau est rasé.

\section{Calchas ait}

Modo piari posse redituras rates

Si placet undas Hectoris sparsi cinis

Ac tumulus imo totus aequetur solo.

Andromaque interprète les deux exigences comme synonymes d'une seule et même profanation :

\section{Prorutus tumulo cinis \\ Mergetur? Ossa fluctibus spargi sinam \\ Disjecta vastis? (648-50)}

On peut se demander toutefois si la menace d'Ulysse est un simple moyen de pression sur Andrornaque ou si les cendres répandues dans les flots n'ont pas une valeur positive, si elles ne pouvaient pas, en effet, servir à piare raies.

Les éléments nous manquent pour répondre à cette question. Nicolas de Damas dans son Ethnôn Synagôgê, disait : «Les gens de Kios brûlent les morts, pilent les os rassemblés dans un mortier ( $\dot{\varepsilon} \nu \delta \mu \omega)$, puis les embarquent avec un crible et prennent la mer. Là ils les éparpillent contre le vent jusqu'à ce que tout soit emporté et disparaisse » (Fr. gr. Hist. 90, fr. 118). Renseignement d'interprétation malaisée. L'holmos est si souvent ustensile de résurrection qu'on chercherait dans cette direction si la suite ne détruisait l'image esquissée.

Selon Aristocratès de Sparte, auteur du II e ou du I er siècle avant J.-C., Lycurgue serait mort en Crète après avoir chargé ses hôtes de disperser ses cendres dans la mer (Plut. Lyc., 31). L'histoire paraît bien une simple réplique au vou de Solon qui, mourant à Chypre, fit répandre ses os tout autour de l'île de Salamine. J'ai tenté de démontrer que lorsqu'on partage un corps royal c'est à la fois pour protéger un territoire et pour resserrer la solidarité de ceux qui l'occupent $\left(^{1}\right)$. Les restes de Solon defendent Salamine. Lycurgue, dit Aristocratès, voulut qu'on dispersât les siens parce qu'il craignait,

(1) Le partage du corps royal, S.M.S.R., t. 34 (1963), fasc. I, pp. 1-25. 
si on les ramenait à Sparte, que ses concitoyens ne changeassent ses lois, sous prétexte qu'il était revenu parmi eux : la même raison qui aurait décidé Solon à quitter Athènes. Nous sommes ici devant un dilemme :

Ou bien l'histoire est fabriquée de toutes pièces, à la fois pour expliquer l'absence à Sparte de tout tombeau du législateur et pour aligner le Spartiate sur l'Athénien.

Ou bien la justification rationaliste seule est tardive, une tradition ancienne, entrée on ne sait comment dans la légende de Lycurgue, mentionnant, pour la Crète, un rite analogue à celui de Kios.

Ce rite servait-il uniquement à disperser des cendres chez un peuple vivant en bordure de la mer - Kios, aujourd'hui Gemlik, est un port sur la côte de Bithynie - ou bien avait-il une valeur positive, les cendres étant offertes à la mer pour la rendre propice aux marins? Le mot d'Ulysse implique cette signification. Une interprétation demanderait des documents plus nombreux, plus sûrs et plus clairs.

Andromaque réagit comme toute Grecque pour qui le mort, inhumé ou brûlé, doit faire retour à la terre. On ne jetait normalement à la mer que les cendres des pharmakoi ou autres disgrâciés rejetés de la communauté.

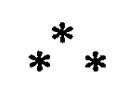

Phèdre s'écarte sur plusieurs points de l'Hippolyte aux couronnes. Thésée est parti pour se rendre aux Enfers, et c'est en se croyant en se souhaitant - veuve que Phèdre se déclare à Hippolyte. Celuici, maudit par son père, ne reparaît plus ; on rapporte sur la scène les débris de son cadavre.

Dans le drame d'Euripide, Thésée couronné, lui aussi, revient d'une théorie et Hippolyte, mortellement blessé, rentre en scène. Sur ces deux points, Racine a suivi Sénèque et non Euripide.

Nous ne pouvons mesurer ce que Sénèque doit à la Phèdre de Sophocle et au premier Hippolyte d'Euripide, celui qui se voilait la face en recevant l'aveu de Phèdre. Ce qui est curieux, c'est que tout ce qui, à nos yeux, lui est propre, restitue l'aspect le plus archaïque de la légende. Son héros est démembré au point que ses fidèles ne peuvent même plus recomposer son cadavre. De même Cadmos, à 
la fin des Bacchantes, recherche dans les buissons les débris de ce que fut Penthée : thème où le sinistre débouche aisément dans le grotesque et que Sénèque a traité avec plus de complaisance que de goût.

Plus d'une légende du héros écartelé vivant existe en Grèce et même à Rome, témoin la fin de Mettius Fufetius. On les a interprétées de plus d'une manière, en marge du totémisme, de l'omophagie, des liturgies de la terre. Vouloir les ramener à une commune mesure serait certainement une erreur. On y distingue les restes d'un scénario rituel qui avait pour centre un sacrifice authentique. Mais à quoi servait celui-ci ? On distingue des composantes agraires, " osiriennes ", dans l'histoire de Lityersès ; Penthée et Lycurgue, doublcts de Dionysos, ne peuvent guère se comprendre en dehors de lui. Le conte de Glaucos est des plus énigmatiques. Il y a toujours, semble$\mathrm{t}$-il, captation de forces, mais non toujours, comme dans le cas d'Osiris, au bénéfice du sol (1). Poursuivre une explication unique fait négliger des singularités qui ont leur prix.

L'histoire d'Hippolyte ne semble contenir aucune composante tellurique. Le myrte de Trézène, dont Phèdre lacère les feuilles dans son obsession amoureuse, a bien l'air d'une invention romanesque (Paus., II, 32, 3). A vrai dire, son culte à Trézène concerne le mariage (Eur. Hipp. 1425) mais non, du moins explicitement, la procréation. Il est toutefois ressuscité par Asclépios pour être identifié ensuite avec Virbius, le premier roi-prêtre de Némi. Ces faits peuvent s'insciire dans un mythe de type osirien, mais ils restent étrangers à tout ce que nous connaissons par les œuvres littéraires.

Euripide en effet et, bien plus clairement, Sénèque, nous ramènent dans une direction toute différente, vers les légendes du prince royal sacrifié pour assurer une victoire à son père ou, davantage, pour prolonger sa vie. Le roi suédois Aun tue l'un après l'autre ses neuf fils afin d'ajouter leurs années à celles qu'il aurait dû vivre normalement $\left({ }^{2}\right)$. Soumise à la vraisemblance, sa légende historicisée sanc-

(1) Voir Mircea Eliade, Traité d'histoire des religions, 1953, p. 129 à 131 ; Angelo BRELICH, Tre variazoni romane sul tema delle orgine, Rome, 1955, p. 125 ; Quirinus, S. M. S. R., 1960 ; M. Delcourt, Partage du corps royal, S. M. S. R., 1963, p. 19.

(2) M, Delcourt, The legend of Sarpedon and the saga of the archer, History of Religions, Chicago, t. II, 1962, p. 46. 
tionne son crime en le faisant vieillir. La mort de chacun de ses fils lui a donné un nouveau délai, mais n'a pas magiquement réparé ses forces, ce qui devait avoir lieu dans la conception primitive, que les exemples réunis par Frazer permettent d'esquisser. Le cours de la nature dépend du roi - «Souveraineté et Fécondité sont des puissances solidaires ", dit Dumézil -, son affaiblissement et la mort naturelle qui s'ensuivraient compromettraient la vigueur de tous. On tue donc le roi quand il commence à décliner parce qu'il ne faut pas que sa maladie se communique à son peuple. D'autre part, on renouvellera périodiquement sa vigueur (par exemple au moment de l'année où tout est ccnsć repartir à zéro) en le supposant mort, puis ressuscité. Pendant son éclipse, un autre prendra sa place - c'est ainsi que Phaéthon se substitue au Soleil - et périra aussitôt, laissant le roi recommencer un nouveau règne avec l'appoint des forces libérées par la mort de la jeune victime.

Le rituel des Sacaea babyloniennes est connu par des auteurs grecs $\left.{ }^{1}\right)$. La fête n'est pas sans analogie avec les Saturnales; et Cronos-Saturne est précisément un dieu qui, comme le roi Aun, a tué ses enfants à mesure qu'ils venaient au monde. Maîtres et serviteurs échangeaient leurs rôles. Un prisonnier condarnné à mort prenait les vêtements du roi et pouvait user de ses concubines, ainsi que le fait Absalon à la mort de David, affirmant ainsi ses droits au trône (II Sam., XVI, 21-23) ( ${ }^{2}$ ). Il était ensuite exécuté après avoir eu, pendant son court règne, tous les attributs de la royauté. L'histoire de Shah Abbas (1586-1628) prouve à quel point la croyance resta vivante: prévenu par ses astrologues que l'année 1591 lui serait maléfique, il abdique et fait occuper le trône trois jours durant par un «infidèle » qui est ensuite exécuté, après avoir déchargé le vrai roi de tout présage défavorable $\left({ }^{3}\right)$.

(1) Dans le IVe discours de Dion Chrys. sur la royauté (ch. 69), Diogène raconte l'histoire à Alexandre pour lui montrer la vanité des attributs royaux. - Strabon ne signale de la fête que l'aspect qui la fait ressembler aux Saturnales (XI, 8,5). - Athénée, d'après Ctésias, fait de même et nomme l'interroi le zoganès sans mentionner sa mise à mort (XIV, 44, p. 639 c).

(2) C'est en épousant le veuve de Laïos qu'Edipe devient roi de Thèbes.

(3) J. G. Frazer, The dying God, pp. 113, 599 et 157-9. Voir l'histoire de Sufi II qui évite les conséquences d'une maladie en prenant un autre nom, Soliman, et en se faisant recouronner. Cette fois le sacrifice du mock-king fut purement symbolique. 
La pièce de Sénèque suit exactement le scénario des Sacaea. Thésée est aux Enfers, considéré comme mort. Il décrit lui-même son lent retour à la vie :

\section{Pars una vitae mansit extincto mihi \\ Sensus (842-3). \\ Heu labor quantus fuit \\ Phlegethonte $a b$ imo petere longinquum aethera \\ Pariterque mortem fugere et Alcidem sequi (847-9).}

L'interrex babylonien s'unit aux concubines: Phèdre s'offre à Hippolyte. Thésée revient et condamne Hippolyte à mort. Le père et lc fils ne se rencontrent pas sur la scène. Le second va. sous terre prendre la place que l'autre a quelque temps occupée. Rien de semblable n'apparaît dans le drame d'Euripide, alors que, dans la Folie d'Héraclès, c'est en revenant de l'Hadès que le héros tue ses enfants qui vont le remplacer chez les morts ( $\left.{ }^{1}\right)$. Racine, qui souvent retrouve des intentions primitives, a dans Phèdre suivi Sénèque et infligé à Thésée une mort temporaire.

On peut se demander pourquoi le sacrifice du prince royal se fait sous la forme d'un démembrement que Sénèque décrit avec une minutie pénible, mais qui appartient bien à la légrende primitive. En effet, la fable 47 de Hygin raconte simplement la tragédie d'Euripide, sauf sur un point, où il dit equi Hippolytum distraxerunt, reproduisant la tradition dominante, car Euripide le décrit simplement entraîné, meurtri contre les rochers où sa tête se heurte, où tout son corps se brise (1237). Il fallait bien renoncer au dépècenent puisque Hippolyte devait être ramené mourant sur le théâtre.

L'importance légendaire du sparagmos, attestée par Sénèque, s'explique peut-être par les vestiges osiriens qui subsistent dans l'histoire d'Hippolyte. Les restes d'Osiris sont enfouis clans la terre; ceux d'Hippolyte sont simplement dispersés à la surface, ce qui suffit pour évoquer l'image des semailles, c'est-à-dire de la résurrec-

(1) De nombreux contes donnent une version censurée du sacrifice du prince royal au profit du roi : pour sauver sa vie, le père expose ou menace celle de son fils. Voir la pénétrante étude de Roland CrAfAY sur L'Eau de Vie et la méthode mythologique des frères Grimm dans Mémoires et publ., de la Soc. des Sciences...du Hainaut, t. 77 (1964), pp. 130-131. 
tion qui attend le héros. Dans les derniers vers de la tragédie, Thésée ordonne aux serviteurs :

Vos apparate regii flammam rogi;

At vos PER AGROS corporis partes vagas

Inquirite. Istam terra defossam premat

Gravisque tellus impio capite incubet.

Hippolyte aura sur le bûcher la consécration supplémentaire du feu. Le cadavre de Phèdre sera écrasé par une glaise trop lourde pour qu'aucune graine y puisse germer.

\section{***}

La Phèdre d'Euripide accuse Hippolyte par une lettre qui est censée expliquer son suicide. Sénèque la met elle-même en présence de Thésée, tenant toujours à la main l'épée dont Hippolyte indigné a voulu la frapper, puis qu'il a laissée tomber. Elle fait de l'épée la preuve de l'attentat qu'elle dénonce.

Cette épée est beaucoup plus qu'un accessoire de tragédie, c'est le symbole parfaitement clair de l'acte que Phèdre appelait de tous ses vœux. Elle marque elle-même les équivalences de l'amour et de la blessure mortelle:

Hippolyte, nunc me compotem voti facis:

Sanas furentem, majus hoc voto meo est,

Salvo ut pudore manibus immoriar tuis (710-2).

«Frappe», dit de même la Phèdre française, et elle lui prend (sans qu'il l'ait menacée) l'arme qu'Enone exhibera comme preuve contre lui. Comme chez Sénèque, le moment où le fer a failli la blesser le sens sexuel de $\tau \iota \tau \varrho \omega ́ \sigma \varkappa \omega$ est bien connu - lui laisse le souvenir d'un affreux délice.

«Hélas, quand son épée allait chercher mon sein,

A-t-il pâli pour moi ? »

(Mais cette épée, c'est elle qui la tenait et non, comme chez Sénèque, l'objet de tant d'anour).

La Phèdre française meurt grâce à « un poison que Médée apporta dans Athènes". Celle de Sénèque se frappe de l'épée fatale, accomplissant elle-même ce qu'Hippolyte a refusé de faire :

Mucrone pectus impium justo patet

Cruorque sancto solvit inferias viro (1196-7). 
Le symbole, qui chez Racine ne fait que passer, domine ici toute la seconde moitié de la pièce.

La Phèdre grecque se pend : seul suicide qui convienne aux femmes atteintes dans leur honneur, Jocaste ou Charila. Comme instrument de suicide, la légende ignore le poison. Le bûcher est réservé aux veuves, aux amantes qui vont rejoindre un homme aimé : Évadné ou Laodamie. Quelques désespérées se jettent dans la mer, comme Ino. Je ne parle là que de l'aspect psychologique de leur acte : ces différentes méthodes correspondent, religieusement, à des croyances différentes. Rarissimes sont les suicides par le fer. Celui d'Ajax est un cas unique. Parmi les femmes, je ne vois guère, en dehors de la Phèdre latine, que Didon: sur le haut du bûcher qui doit détruire son corps, elle se perce de l'épée que lui a donnée Énée (En., IV, 647-65). Philostrate raconte aussi (Heroïca, XX, 17-18) «que Polyxène amoureuse d'Achille vient la nuit sur son tombeau et se penche sur une épée en implorant Achille de lui conserver son

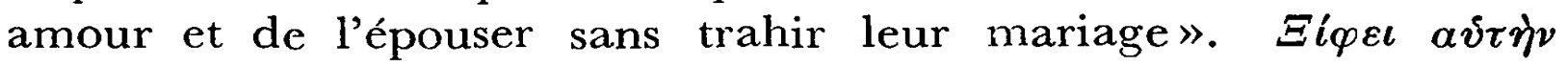
$\varepsilon \dot{\varepsilon} \iota \varkappa \lambda \tilde{\imath} \boldsymbol{\alpha} \iota \iota$ symbolise assez clairement une union.

Le suicide de Phèdre est-il imité de celui de Didon? Je n'en ai pas l'impression. Phèdre, Didon et la Polyxène alexandrine sont toutes les trois des arnoureuses frustrées qui demandent à un symbole de les guérir de leur déception (1). Mais le symbole n'a pas, dans l'Enéide, la force persuasive qu'il a dans la tragédie - même si, dans les deux cas, l'arme mortelle vient de l'homme aimé. Virgile l'a éclipsé par l'image du bûcher royal qu'Énée voit de loin rougeoyer sur Carthage (celui que Thésée refuse à Phèdre) et qui implique, ce qu'il ne dit pas mais que nous savons, la divinisation de Didon.

(1) Pausanias (IV, 2, 7) a trouvé en Messénie une tradition concernant Marpessa, épouse d'Idas, Cléopatra, épouse de Méléagre, Polydora, épouse, d'après les Chants Cypriens, de Protésilaos, une grand-mère, une mère et une fille qui, refusant de survivre à

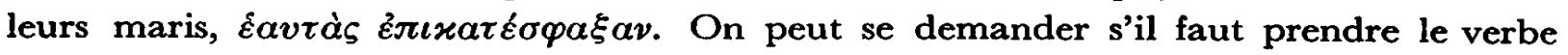
à la lettre pour une époque où la valeur religieuse des différents suicides n'était plus sentie. Ici cependant, il respecte la valeur psychologique. - Celui de Jocaste en revanche, à la fin de l'CEdipe latin, semble bien une simple réplique de celui de Phèdre, justifié uniquement par le goût de Sénèque pour les effusions de sang. 
Le thème de l'épée figurait-il dans une source grecque ? Les tragédies usent parfois d'accessoires - le tapis de pourpre d'Agamemnon, le bouclier d'Hector dans les Troyennes - mais on imagine mal un Hippolyte qui se voile la face esquissant un geste agressif. Hygin (fable 47) dit simplement que Phèdre se pend. Une tradition différente aurait été si extraordinaire qu'il l'aurait probablement mentionnée. Aucun des suicides féminins par le fer, qui ont une seule et même valeur psychologique, ne remonte à la Grèce classique. Cela doit être noté .

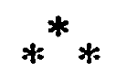

Nous sommes là dans cette marge du domaine religieux où les symboles n'ont plus de correspondances rituelles, étant simplement soutenus par l'inconscient. Portons encore au crédit de Sénèque et de son intuition un détail de Médée.

Pourquoi Médée tue-t-elle ses enfants? La réponse devrait se donner en deux temps.

Au niveau du mythe, un rite corinthien suggère des enfants immolés associés paradoxalement à la figure d'une ancienne déesse guérisseuse.

Au niveau de l'élaboration légendaire, cette Médée devenue magicienne égorge ses enfants pour se venger du mari qui l'abandonne.

Nous n'avons pas à chercher d'où vient le mythe, ni comment il se développa en légende ( $\left.{ }^{1}\right)$, mais de quelle manière, dans une histoire humanisée, les poètes ont justifié l'acte de Médée.

Selon Euripide, elle agirait uniquement pour punir Jason (1050), pour le priver de toute postérité (794-804), raison évidemment insuffisante, mais que Médée ne saurait préciser davantage, car son autre motif, aussi fort que le premier et peut-être davantage, est inconscient. Elle tue pour faire souffrir Jason et, aussi, pour se punir elle-même d'avoir, afin de suivre Jason, assassiné son jeune frère Apsyrtos.

(1) Angelo Brelich, I figli di Medea, S.M.S.R. t. XXX (1959) suppose avec raison un rituel d'initiation. 
Dans la pièce d'Euripide, Jason accepte comme une chose toute naturelle que Créon bannisse ses fils avec leur mère. Chez Sénèque il les garde avec lui, et lorsque Médée les demande afin qu'ils soient les compagnons de son exil, il refuse en alléguant son amour paternel (544). La retouche donne plus de valeur au mobile conscient de Médée.

Ce qui est beaucoup plus intéressant, c'est que Sénèque a amené le mobile inconscient au niveau des idées claires. La Médée d'Euripide a un fort sentiment de culpabilité, mais qui ne se formule pas en besoin d'autopunition. Lorsqu'elle s'accuse d'avoir trahi « son père et sa demeure ", elle évite de nommer Apsyrtos, qui est inclus

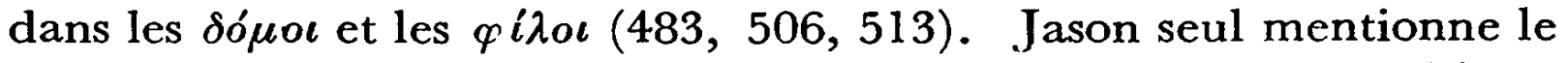
meurtre d'Apsyrtos dans son réquisitoire final (1335), lorsqu'il jette à la tête de Médée les crimes dont il a profité aussi bien que ceux dont il a pâti (1).

La Médée de Sénèque, tout au contraire, n'a pas prononcé quinze vers qu'elle a déjà nommé

$$
\text { divisus ense (131-2); }
$$

Nefandae virginis parvus comes

puis :

$$
\begin{gathered}
\text { Patriumque regnum quaeque fraternus cruor } \\
\text { Perfudit arva (452-3); } \\
\text { Nihil exul tuli } \\
\text { Nisi fratris artus (486-7); } \\
\text { Juvat, juvat rapuisse fraternum caput. } \\
\text { Artus juvat secuisse (911-2). }
\end{gathered}
$$

Mes enfants, dit-elle, sont innocents : et fuit frater (936). Elle pourrait s'arrêter là. Mais Sénèque recule rarement devant l'outrance. Elle voudrait avoir plus d'enfants à immoler : du moins

Fratri patrique quod sat est, peperi duos (957).

(1) Une version de la légende veut que Médée emmène son frère sur le navire Argo puis, sur le point d'être rejointe par son père, tue l'enfant et en jette les membres à la mer pour retarder la poursuite. Je vois dans ce dépècement, qui n'a rien de commun avec celui d'Hippolyte, un sacramentum infanticidii fait pour sceller une association dans le sang. Ainsi le lévite d'Ephraïm groupe les douze territoires d'Israël en partageant entre eux le cadavre d'une femme assassinée (Juges, XIX, 29-30) Cf. M. Delcourt, Partage du corps royal, p. 21. 
Elle hésite encore, mais croit voir l'ombre de son frère qui exige vengeance.

Mihi me relinque et utere hac, frater, manu Quae strinxit ensem (970).

L'enfant qu'elle tue est offert aux mânes de son ancienne victime, pour les apaiser.

Tout cela est si crûment étalé, redit avec une telle lourdeur qu'on en admire Euripide d'avoir laissé le fantôme d'Apsyrtos dans les ténèbres de l'inconscient. Encore faut-il reconnaître que si le trait est trop appuyé, il est juste psychologiquement et même religieusement. Médée a plusieurs raisons d'immoler ses fils; l'une d'elles est de désarmer un jeune mort irrité.

Marie Delgourt. 